\title{
IMPROVING THE SENSITIVITY OF THE OKP VISUAL FIELD SCREENING TEST WITH THE USE OF NEUTRAL DENSITY FILTERS
}

\author{
S. A. VERNON ${ }^{1}$ AND H. A. QUIGLEY ${ }^{2}$ \\ Nottingham and Baltimore, USA
}

\begin{abstract}
SUMMARY
Oculokinetic perimetry (OKP) has been developed to screen for glaucomatous field loss but has relatively poor sensitivity when compared with threshold perimetry.

Forty-two eyes from 42 patients with glaucomatous field loss on Humphrey threshold perimetry and 32 normals performed hand-held OKP under controlled conditions of refraction and lighting. Those who passed the standard test had their OKP cutoff determined with increasing neutral density filters (NDFs) at a new point $15^{\circ}$ from fixation in the inferotemporal field (where first glaucomatous defects are rarest). OKP was then repeated with a NDF that increased the ambient light by $0.3 \mathrm{log}$ units from cut off. Of the $\mathbf{1 6}$ glaucomatous eyes to pass standard OKP, 9 failed the NDF test, improving the sensitivity from $62 \%$ to $83 \%(p<0.05)$. These 9 eyes had field defects that were significantly less severe (mean defect 4.85 vs $7.91(p<0.05)$ and corrected pattern standard deviation 4.12 vs $7.00(p<0.05)$ and were from younger patients (mean age 56 vs 66 years; $p<0.05$ ) than those who failed standard OKP. None of the 32 normals failed standard OKP and only 1 of 32 failed the NDF test. The use of NDFs to customise OKP, producing essentially a staged suprathreshold contrast sensitivity test, appears to increase the sensitivity of the OKP screener without degrading its specificity, particularly in younger subjects.
\end{abstract}

Oculokinetic perimetry $(\mathrm{OKP})^{1}$ has been developed to screen for glaucomatous field loss using a hand-held screener. ${ }^{2}$ In comparative studies with the Humphrey perimeter, the hand-held screener has been shown to a require loss of 16-20 dB compared with age-normal data before defect detection is likely by OKP. ${ }^{3.4}$

Unlike conventional perimetry, OKP uses a black target

From: 'Department of Ophthalmology, University Hospital, Nottingham, UK: 'Dana Centre for Preventive Ophthalmology and Glaucoma Service, Wilmer Institute, Johns Hopkins Hospital, Baltimore, USA.

Correspondence to: MrS. A. Vernon, FRCS, FRCOphth, Department of Ophthalmology, University Hospital, Nottingham NG7 2UH, UK. on a light background. In the hand-held screener the target is $1.5 \mathrm{~mm}$ in diameter, subtending a visual angle of $0.2^{\circ}$ with a high-contrast circular edge. ${ }^{5}$ As this size of target has a normal isoptre of $20^{\circ}$ and targets on the screener are within $15^{\circ}$ of fixation, ${ }^{5}$ OKP effectively produces a suprathreshold, high-contrast stimulus.

In view of the poor sensitivity of OKP in early to moderate glaucomatous defects ${ }^{3.4}$ we investigated whether the use of neutral density filters (NDFs) to reduce the stimulus contrast would result in the detection of earlier defects.

\section{MATERIALS AND METHODS}

Two groups of patients attending the Glaucoma Service of the Wilmer Ophthalmological Institute were recruited to the study. The first were patients with reproducible field defects from glaucoma on at least two consecutive tests with the $30 / 2$ or $24 / 2$ full threshold programme of the Humphrey visual field analyser. Only one eye per patient was studied in this group, eyes being chosen if they displayed relative rather than absolute defects on the Humphrey. All eyes tested had a corrected pattern standard deviation (CPSD) of $>2.4 \mathrm{~dB}$ resulting in a defect at a statistical level of at least $p<5 \%$ (STATPAC 2 analysis).

The second group of patients were ocular hypertensives (intraocular pressure $>21 \mathrm{mmHg}$ and normal discs on biomicroscopy) with normal Humphrey fields (no global indices at $p<10 \%$ on STATPAC 2 analysis) in both eyes on at least two occasions. This group had both eyes tested with the OKP screener. All eyes had a best corrected acuity of at least $6 / 12(20 / 40)$.

All OKP tests were performed in the same office with diffuse overhead illumination from fluorescent units fitted with a diffuser. Reflected light from the OKP chart was measured at 140 lux ( \pm 10 lux) using a light meter. This was checked several times during the course of the study.

For OKP tests, patients wore a trial frame with $25 \mathrm{~mm}$ aperture lenses, with a $2 \frac{1}{2}$ dioptre add over distance cor- 
rection (the OKP test distance is $40 \mathrm{~cm}$ ). The fellow eye was occluded. After a brief explanation of the test and the inbuilt blind spot checks, patients were asked to perform the standard test in a manner previously described. ${ }^{3}$ Only numbers missed on two complete test runs were considered as true defects. A failed test was defined as one or more true defects.

If the patient passed the standard OKP test, NDF OKP was performed. Firstly a 'cutoff' was determined for the eye tested by asking the patient to view a new point (18a) at $15^{\circ}$ eccentricity in the inferotemporal field (see Fig. 1). (This quadrant is least likely to be affected by defects in early glaucoma. ${ }^{6}$ ) Wide-aperture NDFs (TIFFEN, New York; $52 \mathrm{~mm}$ ) of increasing density (0.3 log unit (LU) (3 dB) steps) were then held in front of the trial lens until the central OKP target was just extinguished. This was repeated three times and the mean value taken as the NDF cutoff value. The filter strength was then reduced by $0.3 \mathrm{LU}$ and the OKP test performed as for standard OKP with similar pass/fail criteria. If points were missed, the test was repeated at filter settings 0.6 and then $0.9 \mathrm{LU}$ below the cutoff value. These additional test runs were designed to determine the variation in sensitivity with increasing contrast of the test target. If an eye failed NDF OKP, standard OKP was repeated after a few minutes.

The Humphrey threshold of point $18 \mathrm{a}$ in eyes taking NDF OKP was calculated using the mean of four threshold values equidistant from the relative position of $18 \mathrm{a}$ superimposed on the Humphrey $24 / 2$ or $30 / 2$ program. ${ }^{3}$

\section{RESULTS}

Forty-two patients with glaucoma whose eyes fulfilled the entry criteria took the OKP test. Twenty-six $(62 \%)$ failed and the remaining 16 underwent NDF OKP. Nine of the 16 failed $(56 \%)$. Performing an NDF OKP on eyes that passed standard OKP therefore improved the overall sen-

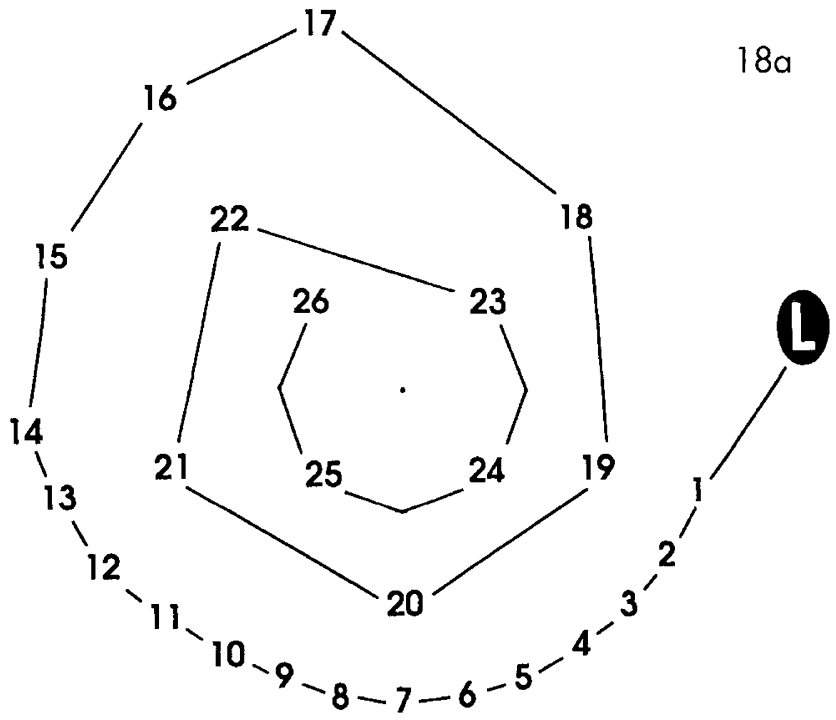

Fig. 1. Hand-held OKP chart designed by Damato using 26 points with an additional point (18a) in the inferotemporal field (left eye).

sitivity of the tests in th:is sample of glaucomatous eyes to $83 \%(p<0.05$ by chi squared). The mean number of test locations missed on NDF OKP at a filter setting $0.3 \mathrm{LU}$ below threshold was 5.6 (SD 4.0).

Six of the 9 eyes to miss points at $0.3 \mathrm{LU}$ also missed points at filter settings 0.6 LU below the NDF cutoff value and 2 of the 9 missed points at 0.9 LU below cutoff. Points missed at less dense NDFs were always missed with filters of greater density. None of the 9 eyes that failed NDF OKP failed the repeated standard OKP.

Eyes that failed standard OKP were from older patients and had significantly greater field defects as measured by both Humphrey parameters than eyes that failed NDF OKP (Table I) (statistics by Mann-Whitney U-test).

Among persons who passed the standard OKP, those

Table I. Comparison of Humphrey mean defect (MD), corrected pattern standard deviation (CPSD) and age in glaucoma eyes failing standard OKP and glaucoma eyes failing OKP with neutral density filters (NDF OKP)

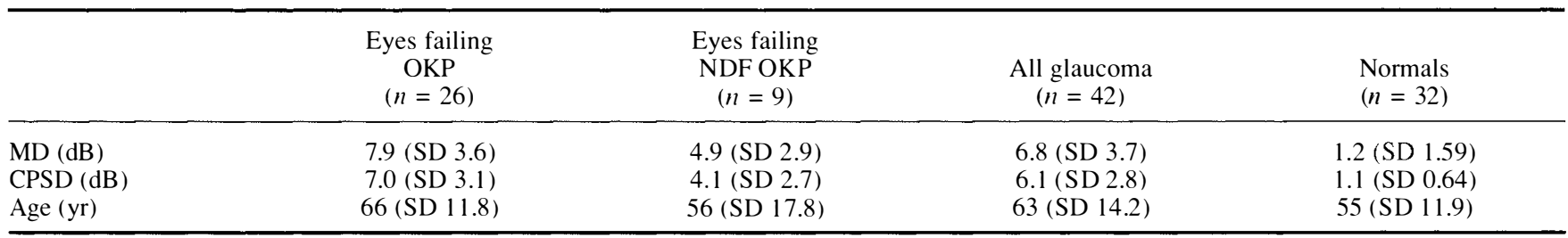

By Mann-Whitney $U$-test, $p<0.05$ for all comparisons (SD, standard deviation). MD negative for glaucomas, positive for normals. Data for all glaucoma and normal eyes also shown.

Table II. Comparison of Humphrey mean defect (MD), corrected pattern standard deviation (CPSD), mean Humphrey threshold point of 18a (see text), neutral density fitter in situ at cutoff (ND CUTOFF) and age in glaucoma eyes failing and passing OKP with neutral density filters (NDF OKP)

\begin{tabular}{|c|c|c|c|}
\hline & $\begin{array}{l}\text { Glaucoma eyes failing } \\
\text { NDF OKP } \\
(n=9)\end{array}$ & & $\begin{array}{c}\text { Glaucoma eyes passing } \\
\text { NDF OKP } \\
(n=7)\end{array}$ \\
\hline CPSD $(\mathrm{dB})$ & $4.1(\mathrm{SD} 2.7)$ & NS & $4.7(\mathrm{SD} 1.6)$ \\
\hline Age $(y r)$ & $56($ SD 17.8$)$ & NS & $62(\mathrm{SD} 16.4)$ \\
\hline Mean Humphrey threshold of $18 \mathrm{a}(\mathrm{dB})$ & $-3.5(\mathrm{SD} 2.5)$ & NS & $-3.1(\mathrm{SD} 6.1)$ \\
\hline NDF CUTOFF (log units) & $2.1(0.41)$ & $p<0.05$ & $1.4(0.57)$ \\
\hline
\end{tabular}

Statistics by Mann-Whitney $U$-test (SD, standard deviation; NS, not significant. 


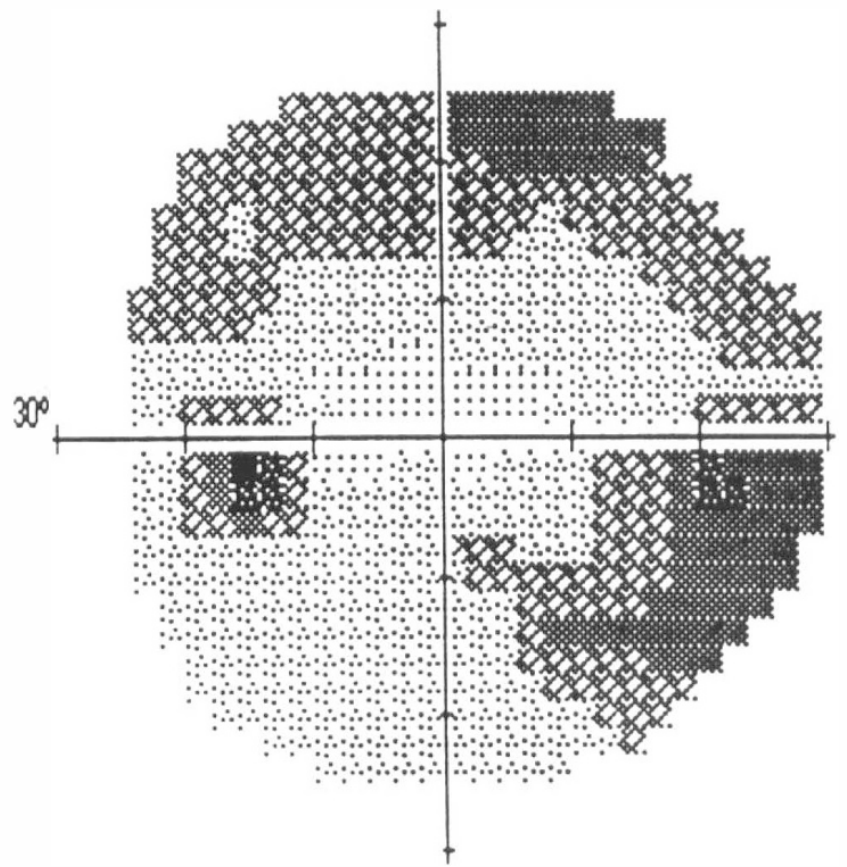

Fig. 2. Humphrey grey-scale of a left eye that passed standard OKP but failed NDF OKP. Humphrey global indices: $M D$, $-6.42 d B(p<0.5)$; PSD, $3.60 \mathrm{~dB}(p<5 \%)$; SF, $1.84 \mathrm{~dB}-$ CPSD, $3.01 \mathrm{~dB}(p<2 \%)$.

who failed NDF OKP required significantly more neutral density at cutoff of point $18 \mathrm{a}$ than those who passed NDF OKP. This was the only difference detected between these two groups (Table II). When the field characteristics of eyes that passed NDF OKP (from Table II) were compared with those which failed standard OKP (from Table I), using the Mann-Whitney U-test, there were again significant differences in both Humphrey parameters $(p<0.05)$.

Humphrey thresholds corresponding to missed NDF OKP points were calculated using a method described previously. ${ }^{3}$ The mean equivalent Humphrey threshold for missed points was $5.78 \mathrm{~dB}$ (SD 1.7) below age-matched normals.

Fig. 2 displays the Humphrey grey-scale and Fig. 3 the NDF OKP result from a left eye with a MD of $-6.42 \mathrm{~dB}$ $(p<0.5 \%)$ and a CPSD of $3.01 \mathrm{~dB}(p<2 \%)$. The 12 points missed on NDF OKP at 0.3 LU below the NDF cutoff of 1.5 LU can be seen to correlate with the Humphrey field defect. On NDF OKP at 0.6 LU below threshold seven points (3-6, 10 and 11) were missed.

Thirty-two ocular hypertensives took both standard OKP and NDF OKP in both eyes. They were similar in age to those glaucoma patients who passed standard OKP (Table I). All 32 passed standard OKP and 31 of 32 passed NDF OKP. One individual failed NDF OKP in one eye on a single point (no. 1).

\section{DISCUSSION}

Undetected glaucoma accounts for approximately $50 \%$ of cases in population-based studies. ${ }^{7-9}$ In order to detect the maximum number of cases when screening, assessment of the visual field is desirable. ${ }^{10}$

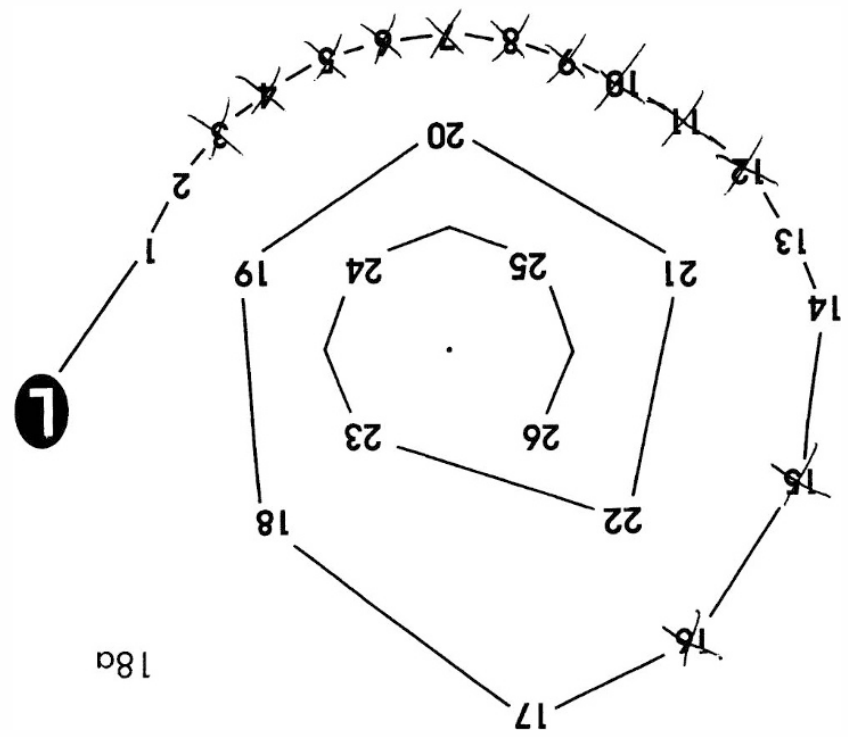

Fig. 3. NDF OKP result with a 1.2 LU NDF in front of the left eye the Humphrey grey-scale of which is shown in Fig. 2. (NDF cutoff was $1.5 \mathrm{LU}$ ). Crosses on the OKP chart indicate repeated misses on two runs. The Chart is inverted for ease of comparison with the Humphrey grey-scale.

The low cost and speed of OKP make it attractive as a screening tool for glaucoma, particularly in remote areas. Its value is determined by its sensitivity and specificity, which will vary depending on a number of factors. These include the pattern of undetected field defects in the particular population to be screened, the prevalence of other disease which the test will detect and the ability of individuals within the population to perform the test. In the United Kingdom and United States the sensitivity of standard OKP when compared with threshold perimetry is unacceptably low unless the aim is to detect only the more advanced defects. As NDF OKP is effectively a suprathreshold contrast sensitivity test, it might be suited to the detection of early glaucomatous defects. ${ }^{11}$

This study has demonstrated that the sensitivity of OKP can be increased with the use of NDFs in a group of glaucoma patients who are experienced in other perimetric techniques. The increase in sensitivity was due to the detection of significantly less severe defects in younger patients that were missed by standard OKP. In $66 \%$ of NDF OKP positive cases a similar defect was detected with a less dense filter in front of the eye. As eyes that failed standard OKP were not tested with NDF OKP, we cannot assume that these eyes would have been detected by the latter test. NDF OKP should therefore be reserved for eyes passing standard OKP until further work has resolved this question.

Interestingly, a number of patients remarked that the low-powered filters $(0.3-0.6 \mathrm{LU})$ increased the visibility of the central test target. It has been shown that a black perimetric stimulus is more stable under varying lighting conditions than a white stimulus, ${ }^{12}$ and although test conditions were designed to reduce glare to a minimum, it is likely that its reduction in these cases explains this phenomenon. 
As there was no difference in field criteria or patient age between those eyes detected by NDF OKP and those missed, why were not all eyes detected? It appears likely that the ability of the patient to see the 18a test target at relatively dense NDFs is important (Table II). As Humphrey thresholds at points corresponding to the $18 \mathrm{a}$ target were not significantly different in those eyes that passed or failed NDF OKP, other factors must determine the ability of an individual to record a high NDF threshold. It is possible that some individuals become 'confused' by the more central points and lines of the spiral between the OKP central target and the fixation point (Fig. 1). Further study on the Nottingham OKP chart, in which a modified circle is used in place of the spiral, will reveal whether an improvement in the sensitivity/specificity ratio is possible.

It is particularly important to detect early defects in the younger age group at risk of glaucoma (40-60 years) if the prevalence of blindness from glaucoma is to be reduced. It is also important that an improvement in sensitivity should not be accompanied by a significant decrease in specificity. Standard OKP in those under 60 years of age has been shown to have a specificity of approximately $99 \%$. NDF OKP in our group of ocular hypertensives (mean age 55 years) achieved a $97 \%$ specificity but does appear to require greater concentration than standard OKP. It is therefore particularly suited to this age group where media opacities and false positives from field defects associated with other conditions such as macular degeneration are rare. Further studies are required to determine more accurately the false positive rate when screening in selected populations.

Key words: Glaucoma, Neutral density filter, Oculokinetic perimetry, Screening, Visual field.

\section{REFERENCES}

1. Damato BE. Oculo-kinetic perimetry: a simple visual field test for use in the community. Br J Ophthalmol 1985;69: 927-31.

2. Damato BE, Chyla J, McClure E, Jay JL, Allan D. A hand held OKP chart for screening of glaucoma. Eye 1990;4: 632-7.

3. Vernon SA, Quigley HA. A comparison of the OKP visual field screening test with the Humphrey field analyser. Eye 1992;6:521-4.

4. Wishart P. Oculokinetic perimetry compared with Humphrey visual field analysis in the detection of glaucomatous field loss. Eye 1993;7:113-21.

5. Mutlukan E., Damato BE, Jay JL. Clinical evaluation of a multi-fixation campimeter for the detection of glaucomatous visual field loss. Br J Ophthalmol 1993;77:332-8.

6. Henson DB, Chauhan BC, Hobley A. Screening for glaucomatous visual field defects: the relationship between sensitivity, specificity and the number of test locations. Ophthalmol Physiol Opt 1988;8:123-7.

7. Hollows FC, Graham PA. Intraocular pressure, glaucoma, and glaucoma suspects in a defined population. $\mathrm{Br} \mathrm{J}$ Ophthalmol 1966;50:570-86.

8. Vernon SA, Hendry DJ, Cater L, Jones SJ. Screening for glaucoma in the community by non-trained staff using semiautomated equipment. Eye 1990;4:89-97.

9. Sommer A, Tielsch JM, Katz J, Quigley HA, Gottsch JD, Javitt J, Singh K. Relationship between intraocular pressure and primary open angle glaucoma in white and black Americans. Arch Ophthalmol 1991;109:1090-5.

10. Tielsch JM, Katz J, Singh J, Quigley HA, Gottsch JD, Javitt J, Sommer A. A population based evaluation of glaucoma screening: the Baltimore eye survey Am J Epidemiol 1991; 134:1102-10.

11. Falco-Reis F, O’Donohue E, Buceti R, Hitchings RA, Arden GB. Peripheral contrast sensitivity in glaucoma and ocular hypertension. Br J Ophthalmol 1990;74:712-6.

12. Mutlukan E, Damato BE. The dark perimetric stimulus. Br J Ophthalmol 1992;76:264-7. 